Length of PICU admission was longer for ex-preterm children (median 4 days) than for those born at term (median 3 days, $p=0.007$ ).

Conclusions: Premature birth places a significant burden on PICU services. Expanding numbers of surviving premature infants combined with the longer length of admission and invasive ventilation requirement are factors to be considered when planning PICU provision to ensure the needs of all children are met.

1241

\section{ANTENATAL STEROIDS IN CHORIOAMNIONITIS: FRIEND OR FOE? A NATIONWIDE SURVEY AMONG PERINATOLOGISTS}

S. Lievense ${ }^{1}$, B.W. Kramer ${ }^{1}$, J.G. Nijhuis ${ }^{2}$, L.J. Zimmermann ${ }^{1}$, J.V. Been ${ }^{1}$

${ }^{1}$ Paediatrics, ${ }^{2}$ Obstetrics, Maastricht University Medical Centre, Maastricht, The Netherlands

Background: Administration of antenatal steroids improves neonatal outcome and is common practice in imminent preterm delivery. Available guidelines delineate chorioamnionitis as a contraindication for antenatal steroids. However, observational evidence suggests antenatal steroids to be safe and effective in chorioamnionitis. We explored perinatologists' opinions on this issue.

Design/Methods: A nationwide email-based survey consisting of four multiple choice questions was performed among all Dutch perinatologists $(n=116)$.

Results: Fifty-nine perinatologists (51\%) sent back a response. In a pregnant woman of 30 weeks gestation with preterm labour and clinical CA, only a minority would actively promote delivery by giving stimulants $(9 \%)$ or performing a C-section (9\%). Fifty-four percent would give antenatal steroids, even though many feel there is a lack of evidence on their efficacy (86\%) and safety $(50 \%)$ in CA. Giving steroids to a mother with clinical CA is considered potentially harmful to either the baby, the mother, or both by $38 \%$. Finally, $64 \%$ of the respondents would favour a clinical trial on the efficacy and safety of antenatal steroids in preterm labour with clinical CA, although another $26 \%$ fear the potential risks for mother and child.

Conclusion: There is great diversity in perinatologists' opinions on the safety and efficacy of antenatal steroids in preterm labour with clinical CA. The majority feel there is lack of evidence on the subject and favour an RCT addressing the issue.

\section{2}

\section{IMPACT OF PANDEMIC H1N1 VIRUS INFECTION IN AN PAEDIATRIC INTENSIVE CARE UNIT}

\section{S. Stabouli ${ }^{1}$, A. Violaki ${ }^{1}$, J. Dotis ${ }^{1}$, M. Mitroudi ${ }^{1}$, S. Kalamitsou ${ }^{1}$, V. Kotsis ${ }^{2}$, M. Kotsiou ${ }^{1}$ \\ ${ }^{1}$ Paediatric Intensive Care Unit, Hippokration General Hospital, Thessaloníki, ${ }^{2} 3$ rd Department of Medicine, Aristotle University of Thessaloniki, Thessaloniki, Greece}

Objective: To describe characteristics and outcomes of paediatric patients in a paediatric intensive care unit (PICU) during $\mathrm{H} 1 \mathrm{~N} 1$ pandemic.

Patients and methods: We included in the study 34 consecutive patients, admitted to the PICU from 01-11-2009 to 28-02-2010. Demographic data, symptoms, comorbid conditions, illness progression, treatments, and clinical outcomes were collected from all patients.

Results: 19 (55.9\%) patients were male and 15 (44.1\%) were female. $29.4 \%$ of the patients were positive for $\mathrm{H} 1 \mathrm{~N} 1$ by viral polymerase chain reaction (PCR) on endobronchial secretions. Subjects with H1N1 infection were older than children who were admitted to the paediatric intensive care unit for other causes $(7.9 \pm 5.1$ years vs. $3.9 \pm 3.7$ years, $P<0.05)$. Duration of intensive care stay and mechanical ventilation was longer in children with $\mathrm{H} 1 \mathrm{~N} 1$ infection $(34.7 \pm 28.7$ vs. $11.8 \pm 17.5$ days, $\mathrm{P}<0.05$ and $32.5 \pm 27.7$ vs. $10.3 \pm 18.6$ days, $\mathrm{P}<$ 0.05 , respectively). $80 \%$ of the children with $\mathrm{H} 1 \mathrm{~N} 1$ infection had known comordid disorders compared to the $33.3 \%$ of the rest of the subjects $(P<0.05)$. Children with $\mathrm{H} 1 \mathrm{~N} 1$ infection presented an increased complications rate $(70 \%$ vs. $16.7 \%, P<0.005)$ and had 4.8 times increased likelihood to present complications compared with the children who were admitted to the paediatric intensive care unit for other causes. Mortality rate was higher in children with H1N1 infection, but the difference between the two groups was not statistically significant.

Conclusions: Critical illness from H1N1 infection in children who require admission to paediatric intensive care unit, was associated with increased complications rate, and prolonged duration of mechanical ventilation and hospitalization. 